\title{
Normal - anders - krank: Die Perspektive des Humangenetikers
}

Wolfram Henn

\section{Formale Kategorien von Normalität}

Eine Betrachtung des mit zahlreichen Konnotationen versehenen Begriffs der Normalität in medizinischer, im Folgenden genauer: genetisch-konstitutioneller Hinsicht erfordert zunächst eine allgemeine semantische Klärung: Was ist unter „normal“ zu verstehen?

Offensichtlich ist das Verständnis von Normalität für einen Mathematiker ein anderes als für einen Juristen und auch innerhalb der Medizin für einen klinischen Chemiker ein anderes als für einen Psychiater. Innerhalb solcher fachspezifischer Kontexte mag es objektive Definitionen oder empirisch begründete Konventionen geben, im umgangssprachlichen Verständnis ist das weitaus weniger der Fall. Hier wird Normalität in aller Regel positiv bewertet; Normabweichungen werden fast immer als Verfehlungen aufgefasst. „Der ist doch nicht normal!“ heißt im üblichen Sprachgebrauch soviel wie „Dieser Mensch ist psychisch auffällig“. Umgekehrt existieren in der Gesellschaft aber auch Einstellungen mit elitärem oder aber subkulturellem Anspruch, nach denen es erstrebenswert ist, sich in Erscheinungsbild oder Verhalten möglichst weit außerhalb sozialer Normen zu bewegen.

Unterzieht man den Begriff der Normalität einer genaueren Betrachtung, so lässt er sich in drei formale Kategorien aufspalten, die sich je nach Betrachtungsobjekt mehr oder weniger stark überlappen: statistische, funktionelle und normative Normalität (s. Abb. 1).

- Am objektivsten zu definieren, zumindest in erster Näherung, ist statistische Normalität: Ein Messwert, der innerhalb einer in Standardabweichungen angegebenen Streubreite um die Mitte der Grundgesamtheit 
liegt, wird als normal definiert. Allerdings kann bereits die Zuordnung, wieviele Standardabweichungen als Normgrenze gelten, durchaus subjektiv gefärbt sein; man denke an Daten wie Körpergröße oder - noch genauer auszuführen - Intelligenzquotienten.

- Hiervon abgeleitet, aber nicht immer deckungsgleich ist funktionelle Normalität. Danach ist eine Eigenschaft solange als normal anzusehen, wie sie empirisch keine schädlichen Auswirkungen auf das Funktionieren des Gesamtsystems hat. In der Medizin ist dieses Normalitätsverständnis wohl am augenfälligsten in der klinischen Chemie. Hier wird aber auch deutlich, dass es sehr unterschiedliche funktionelle Toleranzgrenzen für Normabweichungen geben kann: Das Doppelte des Durchschnittswertes der gesunden Allgemeinpopulation ist bei Leberenzymen weitaus weniger bedrohlich als beim Serumkalium.

- Am subjektivsten, zugleich aber auch am sozial prägendsten, sind normative Zuordnungen menschlicher Eigenschaften oder Verhaltensweisen. Normativ normal sind Menschen immer dann, wenn sie anderen nicht negativ auffallen, speziell wenn sie nicht aufgrund ihrer Auffälligkeiten von gesellschaftlicher Teilhabe oder Chancen ausgeschlossen werden. ${ }^{1}$ Insbesondere bei psychiatrischen Einordnungen spielen kulturelle Kontexte und sogar individuelle Präferenzen eine wesentliche Rolle: Die Grenzen zwischen Ordnungsliebe und zwanghaftem Verhalten oder zwischen lebhaftem Temperament und Hypomanie sind fließend; verschiedentlich wird sogar behauptet, dass aus monetärem Interesse von Ärzten und Pharmaindustrie ganze Krankheitsbilder „erfunden“ und damit eigentlich gesunde Menschen normativ zu Kranken umgedeutet würden. ${ }^{2}$

Im Gegensatz zur gängigen pejorativen Einordnung von Normabweichungen als „Abnormitäten“ steht der Versuch, sie normativ neutral als „Anderssein“ zu beschreiben. Intuitiv kann dies aber nur bei Besonderheiten gelingen, die unzweifelhaft kein Ausgrenzungspotenzial besitzen, jedoch ist selbst bei objektiv bedeutungslosen sichtbaren Auffälligkeiten wie Linkshändigkeit, Strabismus oder Pigmentmalen der Weg zur Diskriminierung erfahrungsgemäß nicht weit. Umgekehrt ist die Benennung von Krankheit oder Behinderung als „Verschiedenheit“ meist als betonte Stellungnahme gegen die Ausgrenzung betroffener Menschen intendiert, wie an Richard von Weizsäckers Wort „Es ist normal, verschieden zu sein" deutlich wird.

Ein augenfälliges Beispiel für die Schwierigkeit, Normalität konsensfähig zu definieren, und zugleich für das Diskriminierungspotenzial von Abweichungen vom Normbereich ist die Intelligenz. Es ist bereits höchst umstritten, ob die zur Messung von Intelligenz verwendeten IQ-Tests überhaupt valide und frei von kulturellen Einflüssen sein können. ${ }^{3}$ Selbst wenn man dies bejaht, stellt sich zunächst für die statistische Normdefinition die Frage nach

\footnotetext{
Hoedemakers/ten Have (1999).

Blech (2003).

Sternberg/Grigorenko (2004).
} 
der Breite des Normbereiches. Wo liegen die Grenzen zwischen Normal-IQ und Hochbegabung auf der einen beziehungsweise Lernbehinderung auf der anderen Seite? Für ein Kind im Vorschulalter hängen seine Bildungschancen davon ab, mit welchen Verfahren bei einer kinderpsychologischen Eignungstestung sein Intelligenzquotient gemessen und auf welche Normalpopulation er bezogen wird.

Die funktionelle Wertigkeit von Intelligenz hängt ganz von den situativen Anforderungen ab. Einfache Aufforderungen kann auch ein Mensch mit recht ausgeprägten mentalen Defiziten adäquat ausführen, wohingegen bestimmte extreme Intelligenzleistungen nur von wenigen Personen überhaupt bewältigt werden können: Auf einem Mathematikerkongress beispielsweise genügt auch ein über dem statistischen Mittel liegender allgemeiner IQ kaum, den Vorträgen intellektuell zu folgen. Mithin kann jeder Mensch mehr oder weniger oft in Situationen geraten, in denen seine kognitiven Fähigkeiten funktionell unzureichend sind, was er dann meist als beschämend empfindet.

Normativ kann die Grenze zur - zweifellos unmittelbar diskriminierungsträchtigen - Minderbegabung weitgehend beliebig nach milieuspezifischen Gepflogenheiten gezogen werden; eine Kategorisierung eines Menschen als „geistig behindert“ kommt seinem weitgehenden Ausschluss von gesellschaftlicher Anerkennung und Teilhabe gleich. Eine statistisch gleich starke Normabweichung der Intelligenz nach oben wird dagegen normativ akzeptiert; allenfalls kann sie ihren Träger über subtile soziale Mechanismen als „Sonderling" ausgrenzen.

funktionell:

Ausführen von Befehlen $I Q>50$

Soziale Kommunikation $I Q>80$

Akademische Karriere $I Q>120$

Lösung Fermat-Theorem $I Q>200$ statistisch:

IQ $75-125$

$(80-130 ; \ldots ?)$

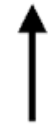

Formale Kategorien von Normalität:

Beispiel Intelligenz

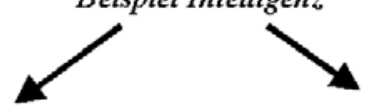

normativ:

milieuspezifisch

$I Q>80$ ?

$I Q>100 ; \ldots ?$

Abb. 1 Formale Kategorien von Normalität am Beispiel der Intelligenz

\section{Kategorien genetischer Normalität}

Auch auf genetische Merkmale ist die dreigliedrige Einteilung von Normalität anwendbar, allerdings mit gewissen Besonderheiten: In Betracht zu ziehen sind lediglich die etwa $10 \%$ des menschlichen Genoms, die für im Phänotyp 
ausgeprägte Merkmale codieren. Varianten in nicht-codierenden Bereichen des Genoms führen definitionsgemäß nicht zur Manifestation von phänotypischen Eigenschaften oder gar Krankheiten; individuelle Bedeutung können sie allenfalls als Ergebnisse von Laboranalysen im Zusammenhang von forensischen Untersuchungen oder Vaterschaftstests erlangen.

Von zentraler Bedeutung für genetische Normzuordnungen sind die Begriffe „Polymorphismus“ und „Mutation“. Nach einer gängigen formalgenetischen Definition bezeichnen Polymorphismen häufige genetische Varianten, die in mindestens $1 \%$ der Allgemeinbevölkerung verbreitet sind, Mutationen dagegen seltenere Varianten. Allerdings ist diese Einteilung für die klinische Praxis und auch die ethische Betrachtung wenig tauglich, weil es durchaus Varianten im Genom gibt, die zwar statistisch selten sind, aber auf den Phänotyp des Individuums nur so geringe Auswirkungen haben, dass sie weder funktionell noch normativ bedeutsam werden können. Für die hier anzustellenden Überlegungen brauchbarer ist die klinisch-genetische Definition, nach der unter Polymorphismen, unabhängig von ihrer Häufigkeit, genetische Varianten ohne Krankheitswert und unter Mutationen solche mit Krankheitswert verstanden werden.

Ein bekanntes Beispiel für genetische Polymorphismen sind die Blutgruppen des ABo- und des Rhesus-Systems, für die es zwar innerhalb der gesunden Allgemeinpopulation verschiedene messbare Varianten gibt, die aber außerhalb besonderer Konstellationen wie etwa einer Rhesus-Inkompatibilität zwischen Schwangerer und Fetus keine gesundheitliche Bedeutung haben. Bestimmte Allelkonstellationen im Rhesus-System sind in der Population extrem selten und entsprechen damit der formalen, nicht aber der klinischen Definition einer Mutation. Wohl niemand käme auf die Idee, einen Menschen mit einer seltenen Blutgruppe als „genetisch abnorm“ zur bezeichnen, weil sich sein diesseits von Laborbefunden wahrnehmbarer Phänotyp nicht vom gesellschaftlich Üblichen unterscheidet. Vielmehr kommt hier nach intuitivem Verständnis zwanglos der Begriff des „Andersseins“ zur Anwendung. (Nur am Rande sei bemerkt, dass eine seltene Blutgruppe im Falle einer Transfusionsbedürftigkeit tatsächlich funktionelle Relevanz erlangen kann, dennoch würde sie wohl auch in dieser Konstellation normativ nicht als Defektzustand wahrgenommen werden).

Eine Besonderheit genetischer Merkmale besteht in der Vererbbarkeit rezessiver Krankheitsanlagen, die im Phänotyp nicht ausgeprägt werden. Es ist keineswegs selten, dass ein klinisch völlig gesunder Mensch eine genetische Defektanlage trägt, die durch die vom anderen Elternteil ererbte Normalanlage ausgeglichen wird und somit für ihn selbst keinerlei gesundheitliche Relevanz besitzt, wohl aber möglicherweise für Nachkommen. Ein bekanntes historisches Beispiel ist die englische Königin Victoria, die über sechzig Jahre lang regierte und als ein Musterbeispiel gesundheitlicher Robustheit galt, aber als Trägerin eines überdeckten Defektes im Gen für den Blutgerinnungsfaktor VIII auf einem ihrer beiden X-Chromosomen an männliche Nachkommen in mehreren Generationen die Hämophilie A vererbte. 
Wie sind solche Anlageträgerschaften für rezessive Krankheiten - vermutlich ist jeder Mensch Anlageträger für mehrere rezessive Defektallele - statistisch, funktionell und normativ zu bewerten? Dies sei am Beispiel der in Europa häufigsten rezessiven Erbkrankheit, der Mukoviszidose, erörtert.

Statistisch tragen etwa $95 \%$ der Europäer homozygot, also in beiden von Mutter beziehungsweise Vater ererbten Kopien des Mukoviszidose-Gens das Wildtyp-Allel, also die häufigste und für ein in typischer Weise funktionsfähiges Protein codierende Anlage. Knapp 5\% sind heterozygote Mutationsträger; bei ihnen liegt ein Wildtyp-Allel sowie ein nach klinisch-genetischer Definition mutiertes, also für ein defektes Protein codierendes Allel vor. Etwa jeder zweitausendste Mensch in Europa ist homozygoter Träger von Mutationen in beiden von Mutter beziehungsweise Vater ererbten MukoviszidoseGenkopien. ${ }^{4}$

Funktionell betrachtet sind die homozygoten Wildtyp-Träger wie auch die heterozygoten Mutationsträger in ihren vitalen organischen Funktionen unbeeinträchtigt und nur durch molekulargenetische Analysen voneinander unterscheidbar, denn auch die verbliebene Wildtyp-Genkopie des heterozygoten Mutationsträgers reicht zur Bildung einer hinreichenden Menge funktionsfähigen Genproduktes aus. Anders ausgedrückt ist die klinisch manifeste - in ihrem Schweregrad höchst variable - Mukoviszidose-Krankheit des homozygoten Mutationsträgers die einzige funktionell abnorme Konstitution für das Mukoviszidose-Gen. Dies gilt allerdings nur auf der Ebene des Individuums, da das Zusammentreffen eines heterozygoten Mannes mit einer heterozygoten Frau trotz der jeweils unbeeinträchtigten eigenen organischen Gesundheit für jeden gemeinsamen Nachkommen ein Risiko von 25 \% für eine homozygote und damit klinisch ausgeprägte Mukoviszidose bedeutet. Dabei ist es ein reiner Zufallseffekt, ob das Elternpaar überhaupt letztlich ein oder mehrere kranke Kinder bekommt; angesichts der geringen durchschnittlichen Kinderzahl in Europa bleibt die Mehrzahl der „Risikoehen“ unentdeckt.

An dieser Stelle wird erkennbar, dass genetische und klinische Kategorien von Normalität erheblich divergieren können, da sich genetische Normabweichungen phänotypisch nicht bemerkbar machen müssen und, falls es doch geschieht, die Manifestation der Anomalie sich auch in einem anderen Individuum, nämlich einem Nachkommen, vollziehen kann.

Daraus ergibt sich eine Unschärfe in der normativen Zuordnung: Nach intuitiven Maßstäben, die sich am Phänotyp orientieren, ist ein homozygoter Wildtyp-Träger zweifellos normal und ein homozygoter, klinisch manifester Mutationsträger ebenso zweifellos klinisch krank und genetisch abnorm. Der Heterozygote, der selbst nicht krank ist und nur in bestimmten zufallsassoziierten Konstellationen kranke Kinder haben kann, entzieht sich hergebrachten normativen Einordnungen von Normalität (s. Abb. 2).

4 Tümmler/Stuhrmann (2003). 


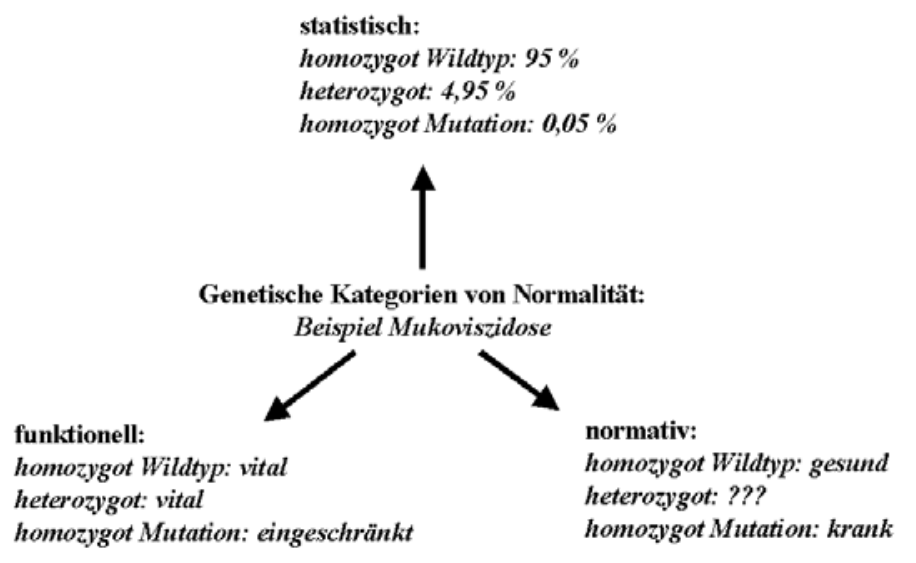

Abb. 2 Kategorien genetischer Normalität: Beispiel Mukoviszidose als rezessives Erbleiden

\section{Der klinische und der genetische Anomaliebegriff}

Damit sind wir bei der Frage angekommen, welche genetischen Varianten, die zwischen klinisch unauffälligem Wildtyp und klinisch als Krankheit manifestierter Mutation liegen, zu Zwischenzuständen führen, die normativen Intuitionen von Normalität nicht zugänglich sind, und welche Konsequenzen dies für die betroffenen Menschen hat.

Hier sind verschiedene Konstellationen möglich und auch keineswegs selten, deren normative Zuordnung innerhalb oder außerhalb medizinischer bzw. sozialer Normen schwerwiegende Folgen im Sinne von „genetischer Diskiminierung" haben kann.

Der heterozygote Anlageträger

Bereits beschrieben wurde der Status der überdeckten Anlageträgerschaft eines phänotypisch völlig gesunden Menschen für ein rezessives Erbleiden. Für seine eigene Gesundheit und Lebenserwartung ist dieser - statistisch wie erwähnt ohnehin normale - Zustand zunächst ohne Belang; auch ein Arbeitgeber oder der Anbieter einer Berufsunfähigkeitsversicherung würde hiervon keine Notiz nehmen wollen.

Problematisch wird ein solcher Status aber im Zusammenhang mit der Fortpflanzung. Von dem Moment an, in dem ein Elternpaar erfährt, dass ein gemeinsames Kind ein rezessives Erbleiden hat, schwebt über ihm das Damoklesschwert des 25\%igen Wiederholungsrisikos für dieselbe Krankheit bei weiteren gemeinsamen Nachkommen. ${ }^{5}$

Damit wird jede weitere Schwangerschaft definitionsgemäß zur Hochrisikoschwangerschaft, und der soziale Druck auf diese Paare, dann eine Pränataldiagnostik mit der Option eines Schwangerschaftsabbruchs in Anspruch zu nehmen, ist erfahrungsgemäß hoch. Der bewusste Verzicht hierauf wird in

5 Grabowski (2000). 
der Gesellschaft vielfach als verantwortungslos und die Geburt eines zweiten behinderten Kindes entsprechend als schuldhaftes Fehlverhalten der Eltern angesehen. ${ }^{6}$ Es hat sogar bereits eine Diskussion darüber begonnen, inwieweit in diesem Sinne „vermeidbare“ kranke Kinder von Leistungen privater Krankenversicherungen ausgeschlossen werden dürfen. ${ }^{7}$

Durch die seit etwa fünfzehn Jahren bestehende Möglichkeit, mittels genetischer Tests Heterozygote zu identifizieren, bevor sie kranke Kinder bekommen, sind sie zum Objekt angewandter negativer Eugenik durch das sogenannte prämaritale Heterozygotenscreening geworden. In Ländern wie Zypern oder Israel, in denen eine besonders hohe Heterozygotenrate für bestimmte rezessive Erbleiden besteht, gehört das Screening Heiratswilliger bereits zum gesellschaftlichen Standard; wer sich weigert teilzunehmen, muss mit Nachteilen auf dem Heiratsmarkt rechnen. ${ }^{8}$ Vor dem Hintergrund historischer Belastungen besteht hierzulande dagegen eine verständliche Zurückhaltung gegenüber dem Heterozygotenscreening. ${ }^{9}$

\section{Der „Noch-nicht-Kranke“}

Nicht jede Erbkrankheit manifestiert sich bereits zum Zeitpunkt der Geburt, vielmehr sind genetische Krankheitsanlagen ihrem Wesen nach zeitlich von der organischen Ausprägung der Krankheit entkoppelt. Das bekannteste Beispiel dafür ist die Huntington-Krankheit, ein dominantes Erbleiden, das sich bei Anlageträgern nach zuvor unbeeinträchtigter organischer Gesundheit meist erst jenseits des dreißigsten Lebensjahres manifestiert, dann aber unaufhaltsam bis zum Tode fortschreitet. Viel häufiger sind erbliche Krebserkrankungen; mehrere hunderttausend Menschen allein in Deutschland sind Anlageträger für erblichen Darmkrebs oder Brustkrebs. Für sie gilt ein extrem hohes, wenn auch meist nicht 100\%iges Risiko, im Lauf des Lebens an Krebs zu erkranken..$^{10}$

Mit der Identifikation verantwortlicher Gene ist die prädiktive genetische Diagnostik zur Realität geworden: Ein Nachkomme eines von einer spätmanifestierenden dominanten Krankheit betroffenen Elternteils trägt ein A-prioriRisiko von je $50 \%$, die Krankheitsanlage geerbt zu haben oder nicht. Mit einer molekulargenetischen Blutuntersuchung besteht nun die Möglichkeit, dieses Risiko im Sinne eines sicheren Ausschlusses oder aber des definitiven Nachweises der Anlageträgerschaft zu präzisieren. ${ }^{11}$ Ein Mensch, der durch einen solchen prädiktiven Gentest als Anlageträger für ein spätmanifestierendes Erbleiden identifiziert worden ist, weiß also bei der Huntington-Krankheit mit absoluter Sicherheit, bei erblichen Krebserkrankungen mit hoher Wahrscheinlichkeit, dass ihm eine schwere Krankheit bevorsteht. Er ist zwar zum Zeitpunkt des Tests meist organisch gesund, wird durch dieses Wissen aber

\footnotetext{
6 Lenhard et al. (2005).

7 Henn (2007).

8 Broide et al. (1993).

9 Bundesärztekammer (1992).

10 Lynch/Fusaro/Lynch (1997).

11 Benjamin et al. (1994).
} 
zum „Noch-nicht-Kranken“ oder zum „Unpatienten“. ${ }^{12}$ Während für die Huntington-Krankheit keine medizinische Prävention, sondern lediglich eine Anpassung der Lebensplanung an das unausweichliche Schicksal möglich ist, besteht für Anlageträger erblicher Krebserkrankungen die Indikation zu engmaschigen Vorsorgeuntersuchungen, bei bestimmten Krankheiten wie etwa der familiären Darmpolyposis sogar die einzige lebensrettende Notwendigkeit einer präventiven Colektomie. Zweifellos entzieht sich der gesundheitliche Status einer zwanzig Jahre alten, körperlich völlig gesunden Frau mit molekulargenetisch nachgewiesener Anlage für Darmpolyposis allen hergebrachten Kategorien von Gesundheit, Anomalie oder Krankheit. Entsprechend kontraintuitiv und emotional schwierig ist es sowohl für die Patientin selbst als auch den behandelnden Chirurgen, einen zu diesem Zeitpunkt problemlos funktionierenden und tumorfreien Dickdarm komplett zu entfernen.

Wie keine andere Gruppierung in der Gesellschaft sehen sich die prädiktiv identifizierten oder auch nur familienanamnestisch als Risikoperson für ein spätmanifestierendes Erbleiden erkennbaren „Noch-Nicht-Kranken“ der Gefahr einer Diskriminierung aufgrund ihres genetischen Status gegenüber. Sowohl Arbeitgeber wie auch private Versicherungsunternehmen haben ein starkes Interesse daran, dass die sich bei ihnen um einen Vertrag bemühenden Personen nicht mit verdeckten gesundheitlichen Belastungen behaftet sind. Hier kann auch für einen nach allen klinischen Kriterien vollständig gesunden Menschen sein abnormer Genotyp durch normative Bewertung unmittelbar in die soziale Ausgrenzung und zu materiellen Nachteilen führen, selbst dann, wenn bei ihm die tatsächliche künftige Manifestation der Krankheit nicht feststeht. Dementsprechend legen Betroffenenorganisationen äußersten Wert auf zurückhaltenden Umgang mit prädiktiv bedeutsamen genetischen Daten, und rechtliche Schutzmechanismen werden inzwischen von politischer Seite entwickelt. ${ }^{13}$

\section{Der multifaktorielle Risikoträger}

Über die seltenen, künftige Krankheiten weitgehend unausweichlich vorherbestimmenden monogenen Krankheitsanlagen hinaus gibt es auch genetische Dispositionen für in der Bevölkerung häufige multifaktorielle „Volkskrankheiten“. Allerdings werden die zugrunde liegenden genetischen „Suszeptibilitätsfaktoren“ in ihren Auswirkungen von exogenen Einflüssen, darunter auch willentlich steuerbaren Verhaltensweisen, modifiziert. So trägt etwa jeder fünfzehnte Europäer eine als „Faktor-V-Leiden“ bezeichnete Variante in der DNA-Sequenz des Gens für ein Protein im Blutgerinnungssystem. Sie führt zu einem statistisch etwa siebenfach erhöhten Risiko, im Lauf des Lebens an einer Venenthrombose zu erkranken. Nimmt eine Faktor-V-Leiden-Trägerin orale Kontrazeptiva ein, erhöht sich das Thromboserisiko auf das Dreißigfache; raucht sie, ist sie übergewichtig oder treibt keinen Sport, nimmt das Risiko immer weiter zu. ${ }^{14}$ In ähnlicher Weise gibt es eine Vielzahl von in der

12 Rautenstrauch (2003).

13 IHA/WFN (1994); Henn (2005).

14 Vandenbroucke et al. (1996). 
Allgemeinbevölkerung häufigen genetischen Suszeptibilitätsfaktoren für Diabetes, Hypertonie, Altersdemenz und andere nach landläufigem Verständnis nicht erbliche, allenfalls mitunter „in der Familie liegende“ Krankheiten. Angesichts der großen Zahl und der hohen relativen Häufigkeit dieser genetischen Risikoanlagen ist zweifellos jeder Mensch nach seiner genetischen Ausstattung für bestimmte Krankheiten anfälliger, gegen andere resistenter als der Bevölkerungsdurchschnitt. Folglich ist auch jeder Mensch bezüglich seines individuellen Risikos für bestimmte Krankheiten statistisch, bei Vorliegen entsprechender exogener Risikofaktoren auch funktionell abnorm. ${ }^{15}$ Normative Bedeutung im Sinne von Diskriminierungspotenzial ergibt sich daraus aber in aller Regel nicht, da sich in arbeits- oder versicherungsmedizinischer Hinsicht über das gesamte Spektrum möglicher multifaktorieller Krankheiten hinweg meist keine relevanten interindividuellen Risikounterschiede errechnen lassen. ${ }^{16}$

Allerdings gibt es in der Arbeitsmedizin zunehmende Tendenzen, genetische Untersuchungen auf im üblichen Leben bedeutungslose Stoffwechselvarianten in Eignungsuntersuchungen für bestimmte, beispielsweise mit definierten Schadstoffexpositionen belastete Arbeitsplätze einzubeziehen. ${ }^{17}$ Hier kann es einem sich zuvor für „genetisch normal“ haltenden Arbeitnehmer widerfahren, durch den Betriebsarzt aufgrund einer zunächst nur statistischen, nur in einem bestimmten Umfeld als funktionell bedeutsam drohenden, funktionellen Anomalie normativ ausgegrenzt zu werden. Es lässt sich trefflich darüber streiten, ob diese Form der genetischen Diskriminierung nicht doch letztlich in seinem eigenen besten Interesse ist. Ganz neu ist diese Situation übrigens nicht: Schon lange gehört die Überprüfung der Farbsehtüchtigleit mit Ishihara-Zahlentafeln - sie ist nichts anderes als eine genetische Untersuchung auf Phänotypebene - zum Standardrepertoire von Einstellungsuntersuchungen.

\section{Der stolze Andersartige}

„Say it loud, I'm black and I'm proud“: ${ }^{18}$ Aus der amerikanischen Bürgerrechtsbewegung der sechziger Jahre hat sich in der Folge eine Kultur selbstbewusster Zugehörigkeit zu Minderheiten entwickelt, aus der nach Black Power über Gay Pride auch eine weltweite Behindertenbewegung entstanden ist. Über das Streben nach rechtlicher Gleichstellung und gesellschaftliche Teilhabe hinaus hat sich dabei verschiedentlich eine Tendenz entwickelt, das eigene, nach landläufiger Meinung als Defekt verstandene gesundheitliche Merkmal nicht nur als wertneutrale Erscheinungsform menschlicher Vielfalt, sondern sogar als positiv identitätsstiftende und die statistische Mehrheit der Menschen ausgrenzende Andersartigkeit zu begreifen.

Schon immer haben sozial stigmatisierende beziehungsweise funktionell die Lebensgestaltung prägende Anomalien ihre Träger zusammengeführt,

\footnotetext{
15 Henn (2001).

16 Knudsen/Loft/Autrup (2001).

17 McCunney (2002).

18 Brown (1968).
} 
um unter ihresgleichen Normalität im geschlossenen Kreis erfahren zu können. Vereinigungen wie etwa der Klub Langer Menschen e.V. verstehen sich nicht mehr bloß als Selbsthilfegruppe zur Bewältigung medizinischer Probleme, sondern auch als im Wortsinne exklusive, nämlich Nicht-Merkmalsträger per Statut ausschließende Vereinigung mit sozialem und kulturellem Anspruch. ${ }^{19}$

Noch einen Schritt weiter geht die „Deaf Nation“. Sie versteht Taubheit mit „Deaf“ in Großschreibung als Erkennungszeichen - nicht als Behinderung, sondern als verbindendes Merkmal einer volksähnlichen Gemeinschaft, die eine eigenständige Sprache und Kultur hervorgebracht hat, bis hin zu einer eigenen Universität - Gallaudet University -, eigenen „Deaflympics“ und sogar „SuperDeafy“, einer tauben Superhero-Figur. ${ }^{20}$ Die „Culturally Deaf“, wie sie sich selbst bezeichnen, verstehen ihre Gebärdensprache als der gesprochenen Sprache gleichwertige alternative Kommunikationsweise; konsequent werden von ihnen Menschen, die der Gebärdensprache nicht mächtig sind, analog zur für sie selbst gängigen Bezeichnung „hearing impaired“ als „signing impaired" bezeichnet.

Hier wird also in betont präziser Weise statistische und im allgemeinen Lebenskontext auch funktionelle Abnormität zu einer alternativen Normalität umgedeutet, die im selbstgewählten eigenen Umfeld - der Begriff „Parallelgesellschaft" drängt sich auf - für die außerhalb stehende Bevölkerungsmehrheit einen Defizienzstatus begründet.

Diese Haltung mag man als konsequent gelebtes Anderssein bewundern oder auch als skurriles Sektierertum belächeln: Auch an ihrem Ende steht die Diskriminierung der als normativ abnorm definierten Mehrheit, hier durch durch „pro-disability selection“. In einer Umfrage innerhalb der Deaf Community erklärten $29 \%$ der an Pränataldiagnostik für genetisch bedingte Taubheit interessierten Erwachsenen, sie würden ein taubes Kind einem hörenden vorziehen. ${ }^{21}$

Wie auch immer der eigene ethnische, medizinische oder genetische Status auch sein mag: Der dem Menschen wohl evolutionär eingeprägte Drang, nach Möglichkeit den Phänotyp- und Verhaltensnormen der ihn umgebenden Mehrheit zu entsprechen und sich, wenn es denn unvermeidlich ist, allenfalls positiv von der Norm abzuheben, bricht sich immer wieder Bahn. Vielleicht verhilft die Schärfung des Bewusstseins, dass kein Mensch in allen Lebenslagen den jeweils gültigen statistischen, funktionellen und normativen Normen entsprechen kann, am besten zum gelassenen Umgang mit eigenen und fremden Unzulänglichkeiten.

Nobody is perfect.

$19 \operatorname{KLM}(2002)$.

20 Deaf Nation (2007).

21 Middleton/Hewison/Mueller (1998). 


\section{Literatur}

Benjamin et al (1994): Caroline M. Benjamin, Shelin Adam, Sandi Wiggins et al., Proceed with care: direct predictive testing for Huntington disease, American Journal of Human Genetics 55 (1994), p. 606-617

Blech (2003): Jörg Blech, Die Krankheitserfinder. Wie wir zu Patienten gemacht werden, Frankfurt a. M. 2003

Broide et al. (1993): Etty Broide, Marcia Zeigler, Joseph Eckstein, Gideon Bach, Screening for carriers of TaySachs disease in the ultraorthodox Ashkenazi Jewish community in Israel, American Journal of Medical Genetics 47 (1993), p. 213-215

Brown (1968): James Brown, Say it loud, I'm black and I'm proud. Part 1 \& 2, King Records 1968

Bundesärztekammer (1992): Bundesärztekammer, Memorandum: Genetisches Screening, Deutsches Ärzteblatt 89 (1992), S. B1433-B1437

Deaf Nation (2007): About Deaf Nation, www.deafnation.com

Grabowski (2000): Gregory A. Grabowski, Gaucher disease: considerations in prenatal diagnosis, Prenatal Diagnosis 20 (2000), p. 60-62

Henn (2001): Wolfram Henn, Sind wir alle erbkrank?, Universitas 56 (2001), S. 266-274

Henn (2005): Wolfram Henn, Der Diskussionsentwurf des Gendiagnostikgesetzes - Ein Meilenstein der Patientenautonomie? Ethik in der Medizin 17 (2005), S. 34-38

Henn (2007): Wolfram Henn, Auf dem Weg zur „finanziellen Indikation“ zum Schwangerschaftsabbruch bei therapierbaren Erbleiden? Ethik in der Medizin 19, Heft 2 (2007), S. 120-127

Hoedemaekers/Ten Have (1999): Rogeer Hoedemaekers, Henk ten Have, The concept of abnormality in medical genetics, Theoretical Medicine and Bioethics 20 (1999), p. 537-561

IHA/WFN (1994): International Huntington Association, World Federation of Neurology, Guidelines for the molecular genetics predictive test in Huntington's disease, Journal of Medical Genetics 31 (1994), p. 555-559

KLM (2002): Klub Langer Menschen Deutschland e. V., Satzung, www.klub-langer-menschen.de

Knudsen/Loft/Autrup (2001): Lisbeth E. Knudsen, Steffen H. Loft, Herman N. Autrup, Risk assessment: the importance of genetic polymorphisms in man, Mutation Research 482 (2001), p. 83-88

Lenhard et al. (2005): Wolfgang Lenhard, Harald Ebert, H. Joachim Schindelhauer-Deutscher et al., Der Januskopf der Diagnostik: Eltern von Kindern mit Behinderung im Spannungsfeld zwischen Unsicherheit und Ausgrenzung, Geistige Behinderung 44 (2005), S. 99-114

Lynch/Fusaro/Lynch (1997): Henry T. Lynch, Ramon M. Fusaro, Jane F. Lynch, Cancer genetics in the new era of molecular biology, Annals of the New York Academiy of Sciences 833 (1997), p. 1-28

McCunney (2002): Robert J. McCunney, Genetic testing: ethical implications in the workplace, Occupational Medicine-State of the Art Reviews 17 (2002), p. 665-672

Middleton/Hewison/Mueller (1998): Anna Middleton, Jenny Hewison, Robert F. Mueller, Attitudes of deaf adults toward genetic testing for hereditary deafness, American Journal of Human Genetics 163 (1998), p. $1175-1180$

Rautenstrauch (2003): Julia Rautenstrauch, Der Unpatient, Einblick (Zeitschrift des DKFZ) 3 (2003), S. 37

Sternberg/Grigorenko (2004): Robert J. Sternberg, Elena Grigorenko, Intelligence and culture: how culture shapes what intelligence means, and the implications for a science of well-being, Philosophical Transactions of the Royal Society of London, Series B-Biological Sciences 359 (2004), p. 1427-1434

Tümmler/Stuhrmann (2003): Burkhard Tümmler, Manfred Stuhrmann, Molekulargenetische Grundlagen der zystischen Fibrose als Beispiel genetischer Erkrankungen in der Pneumologie, Internist 44 Suppl 1 (2003), S. S7-S15

Vandenbroucke et al. (1996): Jan P. Vandenbroucke, Felix I. M. van der Meer, Frans M. Helmerhorst et al., Factor V Leiden: Should we screen oral contraceptive users and pregnant women?, British Medical Journal 313 (1996), p. 1127-1130 